\title{
Quantitative evidence for neurofilament heavy subunit aggregation in motor neurons of spinal cords of patients with amyotrophic lateral sclerosis
}

D.M.F. Mendonça ${ }^{1,2}$, L. Chimelli ${ }^{2}$ and A.M.B. Martinez ${ }^{1}$

\author{
${ }^{1}$ Departamento de Histologia e Embriologia, Instituto de Ciências Biomédicas, \\ ${ }^{2}$ Serviço de Anatomia Patológica, Hospital Universitário Clementino Fraga Filho, \\ Centro de Ciências da Saúde, Universidade Federal do Rio de Janeiro, \\ Rio de Janeiro, RJ, Brasil
}

\begin{abstract}
Correspondence

A.M.B. Martinez

Departamento de Histologia e

Embriologia, ICB, CCS, UFRJ

Av. Brig. Trompowsky, s/n, Bl. F

21941-540 Rio de Janeiro, RJ

Brasil

E-mail:martinez@ufrj.br

Research supported by FAPERJ,

CNPq, CAPES, and FUJB.

Received July 29, 2004

Accepted March 2, 2005

Amyotrophic lateral sclerosis (ALS), a neurodegenerative disease of unknown etiology, affects motor neurons leading to atrophy of skeletal muscles, paralysis and death. There is evidence for the accumulation of neurofilaments (NF) in motor neurons of the spinal cord in ALS cases. NF are major structural elements of the neuronal cytoskeleton. They play an important role in cell architecture and differentiation and in the determination and maintenance of fiber caliber. They are composed of three different polypeptides: light (NF-L), medium (NF-M) and heavy (NF-H) subunits. In the present study, we performed a morphological and quantitative immunohistochemical analysis to evaluate the accumulation of NF and the presence of each subunit in control and ALS cases. Spinal cords from patients without neurological disease and from ALS patients were obtained at autopsy. In all ALS cases there was a marked loss of motor neurons, besides atrophic neurons and preserved neurons with cytoplasmic inclusions, and extensive gliosis. In control cases, the immunoreaction in the cytoplasm of neurons was weak for phosphorylated NF-H, strong for NF-M and weak for NF-L. In ALS cases, anterior horn neurons showed intense immunoreactivity in focal regions of neuronal perikarya for all subunits, although the difference in the integrated optical density was statistically significant only for NF-H. Furthermore, we also observed dilated axons (spheroids), which were immunopositive for NF-H but negative for NF-M and NF-L. In conclusion, we present qualitative and quantitative evidence of NF-H subunit accumulation in neuronal perikarya and spheroids, which suggests a possible role of this subunit in the pathogenesis of ALS.
\end{abstract}

\section{Introduction}

Amyotrophic lateral sclerosis (ALS) is a progressive neurodegenerative disease of unknown etiology characterized by the loss
Key words

- Amyotrophic lateral

sclerosis

- Spheroids

- Neurofilament proteins

- Immunohistochemistry
...................... 
usually from respiratory failure $(4,5)$, within 2 to 5 years. Up to $50 \%$ of motor neurons may have already degenerated before clinical signs appear. Sensory and cognitive functions are not affected and certain motor neuron groups, including those in the oculomotor and Onuf's nuclei, are also spared (6). The diagnosis is based on the clinical symptoms and/or neuropathologic findings (7). ALS cases can be both sporadic and familial. Five to ten percent of all ALS patients show a family history, and approximately $20 \%$ of them (1-2\% of all ALS cases) have a causative mutation of the $\mathrm{Cu} / \mathrm{Zn}$ superoxide dismutase gene $(8,9)$.

Among the pathologic signs of ALS, hyaline inclusions, ubiquitin positivity and neurofilament (NF) accumulations are prominent features. NF deposits in degenerating motor neurons are considered to be an early pathological hallmark of this disease. These deposits usually involve the anterior horn neurons, both in the soma and in the axonal process. These focal enlargements of neuronal processes, called spheroids, are pale and present crinkled edges when stained with hematoxylin and eosin (10).

$\mathrm{NF}$ are major structural elements of the neuronal cytoskeleton (11) where they play an important role in cell architecture and differentiation, and in the determination and maintenance of fiber caliber (12). They belong to type IV intermediate filaments and are composed of three different polypeptides: light (NF-L), medium (NF-M) and heavy (NF-H) subunits $(13,14)$. Each NF subunit has a 310-amino acid, $\alpha$-helical rod domain following a $\sim 100$-residue head domain, and differences in size are primarily due to the length of the carboxyterminal tail sequences (KSP repeats), which in NF-M and NF-H contain an abundance of charged residues, lysine and glutamic acid, along with numerous serine phosphates $(4,15,16)$. These regions are potential phosphorylation sites (17). Immunohistochemical experiments suggest that the tail domains of NF-M and NF-H are mostly involved in the formation of crossbridges $(12,18)$. NF-H and NF-M subunits are found in the phosphorylated state in normal axons and in the non-phosphorylated state in normal soma and dendrites $(19,20)$.

NF have long been assigned a role in the pathogenesis of several types of neurodegenerative disease, including ALS (4). The abundance of NF in motor neurons may be a natural risk factor for the vulnerability of the motor neurons in ALS (21). In the present study, we performed a morphological and quantitative immunohistochemical analysis to evaluate the presence of each subunit light, medium and high - in spinal cord cell bodies of control and ALS cases and in spheroids of ALS cases.

\section{Material and Methods}

Spinal cords were obtained at autopsy from 3 sporadic ALS patients and from 5 patients without neurological disease (controls). The control patients suffered from one of the following diseases: hypertensive cardiopathy $(\mathrm{N}=1)$, aortic aneurysm $(\mathrm{N}=$ $1)$, acute myocardial infarction $(\mathrm{N}=1)$, and acute respiratory failure $(\mathrm{N}=2)$. The average post-mortem time was $4 \mathrm{~h}$ both in ALS and control cases. The time of fixation was about two weeks. The summary of ALS cases is shown in Table 1. Formalin-fixed, 5- $\mu \mathrm{m}$ paraffin-embedded tissue sections from cervical, thoracic, lumbar, and sacral levels were obtained and collected on gelatin-coated glass slides and stained with hematoxylineosin and luxol fast blue for morphological analysis, or immunostained with the antibodies. ALS cases were then tested for ubiquitin and all were found to be positive. Sections were observed and photographed with a Zeiss Axioskop 2 plus Microscope.

\section{Immunohistochemistry}

Tissue sections were incubated with 3\% hydrogen peroxide in distilled water for 20 
min to block the endogenous peroxidase and then washed three times ( 5 min each) in PBS containing $0.3 \%$ Triton. After incubation for $1 \mathrm{~h}$ with $10 \%$ normal goat serum in PBS$0.3 \%$ Triton, the sections were microwave treated (1 min, twice) in order to expose hidden epitopes and then reacted with a primary antibody overnight at $4^{\circ} \mathrm{C}$. We used the following primary antibodies: anti-NF-H phosphorylated (clone NE14 - 1:40), antiNF-M (clone NN18 - 1:40), and anti-NF-L (clone NR4 - 1:200), all from Sigma (St. Louis, MO, USA). Sections were then incubated with the corresponding biotinylated secondary antibody at a 1/200 dilution (Vector Laboratories, Burlingame, CA, USA) for $2 \mathrm{~h}$. After reaction with the avidin-biotinperoxidase complex (Vectastain ABC Kit, Vector), immunoreactivity was visualized by 5-min exposure to 3,3'-diaminobenzidine/ $\mathrm{H}_{2} \mathrm{O}_{2}$. Primary antibodies were omitted for negative controls. Hematoxylin was used for nuclear staining. Sections were dehydrated in a graded alcohol series, cleared in xylene and coverslipped under permount.

\section{Quantitative analysis}

The immunostaining intensity of NF from ALS and control cases was determined and the material was submitted to careful light microscopic examination for morphological analysis. Thereafter, cell bodies and spheroids were analyzed quantitatively. It is important to emphasize that immunohistochemistry was performed at the same time for all sections used in the quantitative study. Two or three sections of each spinal cord level were used for all ALS and control cases. All neuronal cell bodies found in both anterior horn areas were sampled and all the structures identified as spheroids were also analyzed. The images obtained were captured on an image analysis system and analyzed with the Image Pro Plus software (Media Cybernetics, Baltimore, MD, USA). NF immunostaining was compared quantitatively be- tween ALS and control cases. Because some atrophic neurons exhibited a stronger reaction when compared to normal-sized neurons we evaluated the integrated optical density, which represents the mean optical density multiplied by cell area. "Integrated" refers to the integration, or sum, over a given region, of all the pixel intensity or density values (22). Higher values in the bars represent weaker staining and vice-versa. Statistical analysis was performed by the MannWhitney test using the Prism software (GraphPad Inc., San Diego, CA, USA), with the level of significance set at $\mathrm{P}<0.05$.

\section{Results}

In the present study, we used hematoxylin and eosin to identify the general morphologic aspects of ALS cases and immunohistochemistry to characterize, both qualitatively and quantitatively, the distribution of NF subunits in ALS and control cases, observing each subunit individually.

The normal appearance of motor neurons in the anterior horn of the spinal cord from control cases stained with hematoxylin and eosin can be seen in Figure 1a. The Nissl stain is well distributed throughout the perikarya. The nucleus, when observed, is centrally located. It is possible to observe glial cell nuclei that are lightly stained with hematoxylin. In ALS cases, loss of motor neurons is clearly observed. The remaining preserved neurons presented inclusion bodies with intracytoplasmic organelle displacement (Figure 1b). There was also extensive gliosis

\begin{tabular}{lccc}
$\begin{array}{l}\text { Table 1. Data regarding the amyotrophic lateral } \\
\text { sclerosis cases studied. }\end{array}$ & $\begin{array}{c}\text { Survival time } \\
\text { Sex } \\
\text { after diagnosis } \\
\text { of the disease }\end{array}$ & $\begin{array}{c}\text { Family } \\
\text { history }\end{array}$ \\
\hline M & 65 & 9 months & Negative \\
$M$ & 44 & $\begin{array}{c}\text { 1 year and } 6 \text { months } \\
\text { 1 year and } 5 \text { months }\end{array}$ & $\begin{array}{c}\text { Negative } \\
\text { Negative }\end{array}$ \\
\hline
\end{tabular}




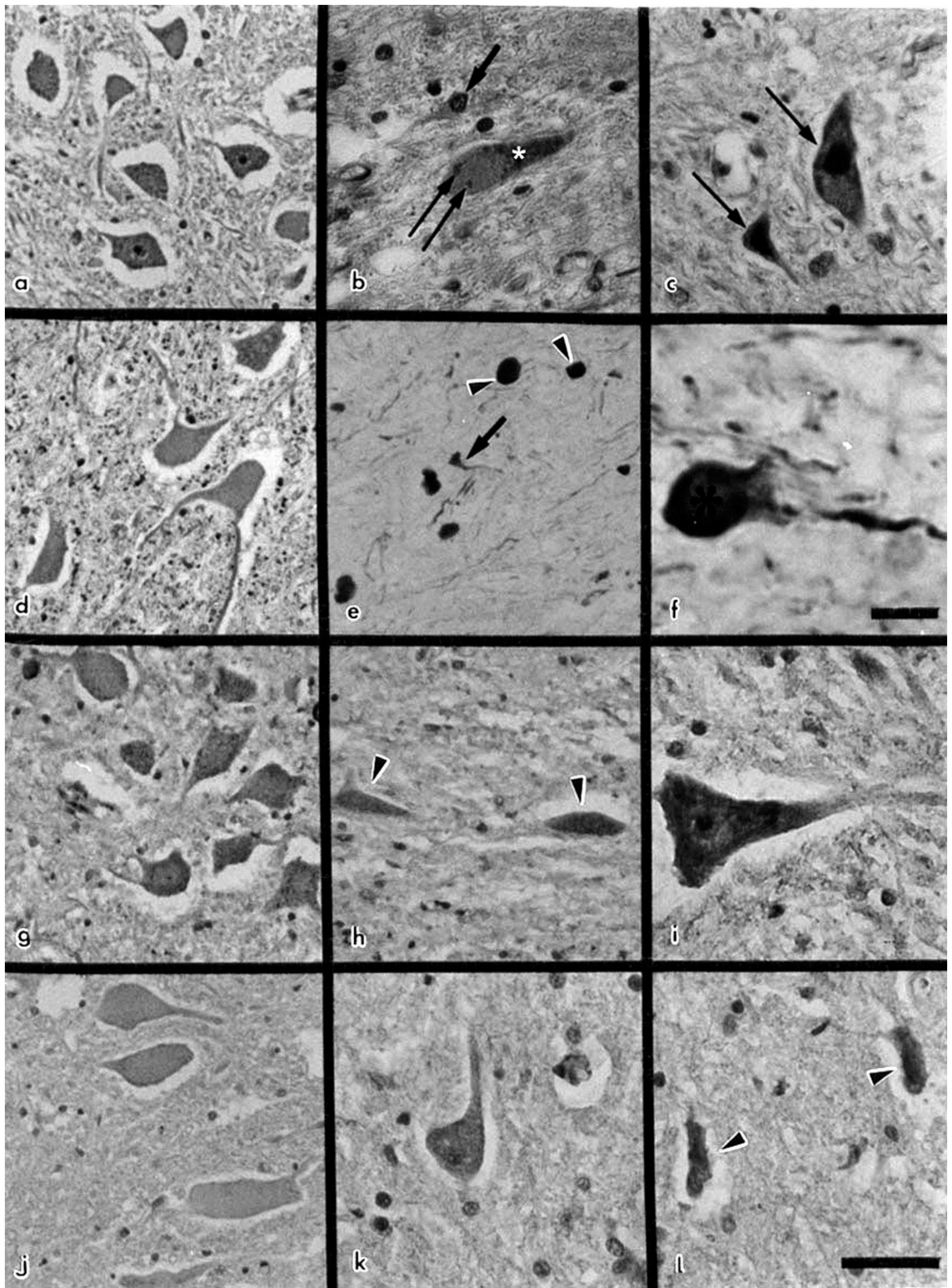


Figure 1. $a-c, H \& E$ staining of ventral horn motor neurons. a, Normal aspect of motor neurons in a control case. All motor neurons display Nissl's stain regularly distributed throughout the perikarya; the nucleus, when observed, is centrally located and glial cell nuclei are lightly stained with hematoxylin. b, $c$, Motor neurons from amyotrophic lateral sclerosis (ALS) cases. b, Motor neuron with intracytoplasmic inclusions (arrows) and Nissl stain displacement (asterisk). Reactive gliosis is also observed (thick arrow). c, Atrophic neuron (arrows). $d-l$, Immunohistochemistry for neurofilaments. $d$-f, Immunohistochemistry for neurofilament heavy subunit (phosphorylated form). $d$, Control case exhibiting weak and equally distributed reaction in the perikarya. $e$, ALS atrophic neuron presenting strong immunostaining (arrow) and spheroids are also observed (arrowheads). $f$, ALS neuronal fragment displaying strong immunostaining (asterisk). g-i, Immunohistochemistry for neurofilament medium subunit. $g$, Motor neurons of a control case exhibiting a strong and uniform immunostaining. $h$, Atrophic neurons presenting stronger immunoreaction (arrowheads). $i$, Motor neuron with strong immunoreaction in perikarya. $j-l$, Immunohistochemistry for neurofilament light subunit. $j$, In control cases the immunoreactivity appears mild and distributed throughout the cytoplasm of motor neurons. $k$, ALS neuron with early morphological alteration exhibiting stronger immunoreaction when compared to control. l, Atrophic neurons presenting strong immunoreaction (arrowheads). Scale bar: (a, b, c, d, e, h, i, k, l) $35 \mu \mathrm{m}$; (g, j) $70 \mu \mathrm{m}$; (f) $10 \mu \mathrm{m}$.

characterized by increased numbers of nuclei and enlargement of cytoplasmic processes (Figure 1b). Some atrophic neurons were also noted (Figure 1c). Neuronal loss was observed at all spinal levels. The lateral corticospinal tract was pale and showed extensive gliosis.

Immunohistochemistry for all tested NF subunits was positive in cell bodies and neurites of controls and in preserved neurons of ALS cases. However, the amount of labeling was different according to the specific subunit and cell region. Immunoreactivity was distributed throughout the cytoplasm and extended into neuronal processes; however, in control cases, cell bodies with the most intense label were those reacting for the NF-M subunit. The axoplasm in the white matter was strongly labeled for most antibodies, except for the NF-L subunit, whose immunoreaction was relatively weak.

\section{Immunohistochemistry for NF-H (phosphorylated form only)}

In control cases, the immunoreactivity for the phosphorylated form of NF-H was weak and equally distributed throughout the perikarya (Figure 1d). However, in the axoplasm of white matter the immunoreactivity was very strong. In ALS cases, some remaining cells presented immunoreactivity similar to control cases, but others presented focal areas of intense staining, indicating an accumulation of the tested subunit in these regions. Atrophic neurons and spheroids were also seen in the ventral horns and showed strong immunostaining (Figure 1e). Structures appearing to be neuronal fragments also showed strong immunostaining (Figure 1f). Spheroids were distinguished from atrophic and fragmented neurons by their rounded appearance and also due to the fact that neurons are not usually rounded and 1 or 2 processes can be seen arising from their cell bodies.

\section{Immunohistochemistry for NF-M}

The antibody to the NF-M epitope labeled neurons in a strong and uniform pattern in control cases (Figure 1g) and the same was observed in white matter axons. In ALS cases, atrophic neurons usually presented a strong immunoreaction when compared to other groups of neurons (Figure 1h). Remaining neurons usually exhibited the same immunoreaction as controls, but some neurons were strongly stained (Figure 1i). Spheroids were negative for this subunit.

\section{Immunohistochemistry for NF-L}

In control cases, the immunoreactivity 
Figure 2. Quantification of immunostaining intensity in cell bodies of control and amyotrophic lateral sclerosis (ALS) cases for all antibodies studied. IOD = integrated optical density. Higher values in the bars represent weaker staining and vice-versa. Data are reported as means \pm SD for 1340 measurements. A, Quantitative comparison of neurofilament heavy (NF-H) subunit immunostaining. Cell bodies of ALS cases were significantly more stained than cell bodies of control cases $(P<$ 0.05, Mann-Whitney test). B, Neurofilament medium (NF-M) subunit and $C$, neurofilament light (NF-L) subunit did not present a significant difference between groups. was mild when compared to the other subunits and regularly distributed throughout the cytoplasm of motor neurons (Figure 1j). Axons in the white matter presented various degrees of staining; some were weakly stained and others were strongly labeled. In ALS cases, immunoreaction seemed to be more intense in preserved neurons and in neurons with early morphological alterations (Figure $1 \mathrm{k}$ ), as well as in atrophic neurons (Figure 11). Similar to NF-M, spheroids did not stain with NF-L.

\section{Quantitative analysis}

Although immunoreactivity for all antibodies used seemed to be more intense in

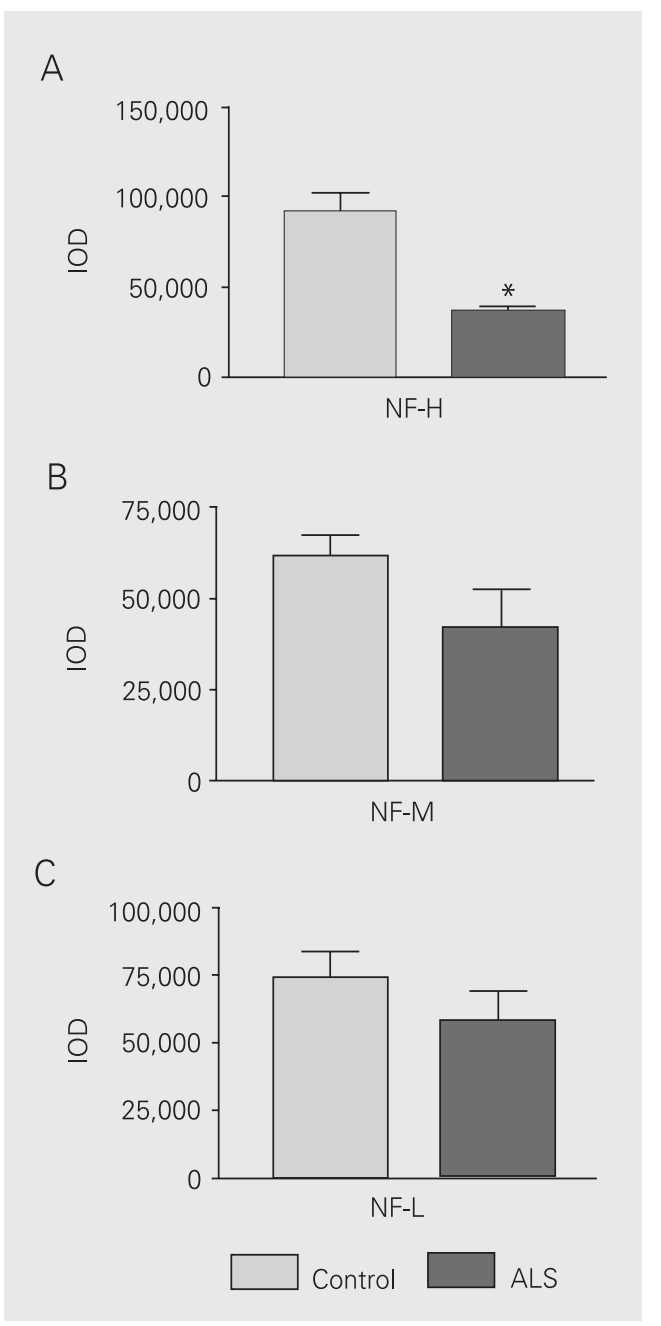

collections of motor neurons from ALS cases when visualized by light microscopy, quantitative analysis revealed that this immunoreactivity was significantly increased only for the NF-H subunit phosphorylated form (Figure 2A). However, for NF-M (Figure 2B) and NF-L (Figure 2C) this increase was not significant.

Spheroids were only positive for NF-H. When we compared immunostaining between spheroids and cell bodies, we observed that they both showed strong immunoreaction with no statistically significant difference (Figure 3).

\section{Discussion}

ALS is a neurodegenerative disease that has been the subject of many studies focusing on different aspects of this disorder. However, the pathogenesis of the disease is still unresolved. An important feature of the pathogenesis of ALS is the presence of NF accumulations in degenerating neurons. The results of the present study show the neuropathological signs of ALS cases and the presence, localization and quantitative distribution of the three NF subunits - NF-L, NF$\mathrm{M}$ and NF-H in control and ALS cases. We also investigated the presence of NFs in swollen axonal processes (spheroids). To our knowledge, our results represent the first quantitative analysis of all different NF subunits in ALS cases, both in cell bodies and spheroids.

In control cases, immunohistochemistry revealed an intense immunoreaction for antibodies against NF-M epitopes in neuronal perikarya. NF-H in its phosphorylated form presented the lightest staining in the perikarya. Both subunits, however, presented a strong staining in neuronal processes. These data are consistent with the fact that the phosphorylated form of NF subunits is mainly located in the axoplasm, while the nonphosphorylated form is found preferentially in the neuronal perikarya and dendrites 
$(19,20)$. The immunoreaction for the NF-L subunit, as described in our results, was discrete in neuronal perikarya and mild to moderate in the processes. This last result agrees with data reported by others $(12,15$, $23,24)$, showing that this subunit is more central to the axis of the filament since it is involved in the arrangement of the core filament, and therefore is less exposed to the antibody.

The classical morphological features of ALS such as dramatic neuronal loss, inclusion bodies associated with intracytoplasmic organelle displacement, atrophic neurons, and extensive gliosis were all present in our cases (6).

The results obtained by immunohistochemistry for ALS cases showed accumulations of immunostaining for all tested antibodies in the perikarya of motor neurons of the ventral horn of the spinal cord. Accumulations of NF proteins, as observed in our cases, can result in abnormalities in the axonal cytoskeleton, leading to a gradual block of axonal transport (4). Various factors may lead to the formation of abnormal accumulations, including dysregulation of intermediate filament gene expression, NF mutations and post-translational modifica-

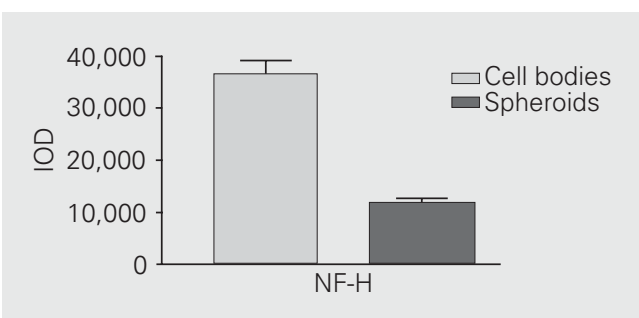

Figure 3. Quantitative analysis of neurofilament heavy (NF-H) subunit on axonal spheroids compared to immunostaining of this subunit in the cell bodies. The difference between the spheroids and cell bodies immunostaining was not significant (Mann-Whitney test) because in this case the comparison was done between amyotrophic lateral sclerosis (ALS) cases; therefore, the sample size was 3 for both groups. In the other comparisons, however, the sample size was the same for ALS cases ( $N=3$ ) but larger $(N=5)$ for control cases. IOD = integrated optical density. tions such as phosphorylation, glycosylation, nitration, and protein cross-linking (25). It is clear from this and previous studies $(11,25)$ that the integrity of the NF network is undoubtedly essential to motor neuron survival and that this network becomes disrupted during motor neuron degeneration. Of particular relevance is the observation that NF inclusions are also found in cases of familial ALS caused by superoxide dismutase mutations, implying a toxic action of NFs in the disease (26).

Statistical analysis of our data confirmed a significant increase of immunoreaction for the antibody used for NF-H, while the increase in NF-M and NF-L was not statistically significant. Our observations indicate that, although all NF subunits seem to be involved in the disease, the NF-H subunit plays a major role in the pathogenesis of ALS. A possible explanation for the nonsignificant results for NF-M and NF-L is the fact that we used only 3 subjects. Thus, if more cases had been available the results for NF-M and NFL might have been significant.

Large neurons, with their fast conducting, large caliber axons require a high content of NFs for structural support and integrity (27). Since NF-rich motor neurons are preferentially affected in human ALS, the quantity of NFs may be important in this selective neuronal vulnerability. Moreover, NF-H plays a major role in the maintenance of fiber caliber since its tail domain is a structural component of cross bridges and is involved in the formation of parallel bundles in NFs (18). In agreement with these observations, Kriz and collaborators (28) reported that transgenic mice expressing high levels of human NF-H proteins present NF inclusions in the perikarya resulting in atrophy of motor axons and altered axonal conductances.

There is growing evidence of a direct participation of NF in many neurological diseases. Hedreen and Koliatsos (29) described in the brains of patients with stroke and other focal lesions the presence of the 
phosphorylated form of NF-H in neuronal perikarya, where it is not normally observed. We have recently observed an increase of NF immunoreaction in degenerating axons of the rat optic nerve following removal of the eyes (30). The present study supports the view that NFs also play a possible role in the pathogenesis of ALS. Furthermore, we present quantitative evidence that the phosphorylated form of the NF-H subunit is the main protein involved in this disease. What is the possible explanation or cause of these observations? We believe that an abnormal phosphorylation of NF-H can induce a cytoskeletal disorder, leading to massive accumulations of hyperphosphorylated protein in the neuronal perikarya and proximal neurites as described by other investigators (31). The literature shows that many factors may account for the formation of NF aggregates in ALS. Recently, it was suggested that glutamate-mediated excitotoxicity, which is one of the factors associated with the pathogenesis of ALS, induces NF side-arm hyperphosphorylation in neurons, leading to an impairment of NF transport (32). Also, in approximately $1 \%$ of sporadic ALS cases there is a mutation in the KSP repeats of the carboxyl terminus of the NF-H gene corresponding to the phosphorylation sites (4). This mutation may also account for the formation of NF inclusions in ALS cases since it was not observed in over 300 normal individuals (4). Another possible cause of NF accumulation and subsequent neurodegeneration in ALS is a failure of the capacity of proteolytic degradation of the aggregates, via the ubiquitin-proteosome pathway, as already described in other neurological diseases $(33,34)$.

Another interesting finding of the present study was the presence of NF-H-positive spheroids in all ALS cases. Spheroids are considered to be a conspicuous and early finding in proximal axons of motor neurons in ALS (35). Although their significance in ALS has not been completely established, they have been described in conditions associated with impairment of axonal transport (36). Our quantitative results showed the presence of phosphorylated NF-H subunits in spheroids. It is possible that hyperphosphorylation increases disassembly of NF thus impairing their axonal transport, as suggested previously (37). A failure of proteolytic degradation might also be associated with accumulation of NF and other intermediate filaments, such as peripherin, in axonal spheroids (35). The presence of phosphorylated NF-H in both the neuronal perikarya and spheroids of ALS cases suggests a possible role of this subunit in the pathogenesis of ALS.

Despite all the evidence presented above, an unresolved question is whether the accumulating NFs are merely by-products of the pathogenic process or active participants in motor neuron dysfunction. However, our observations may contribute to the idea that the high NF content in motor neurons may account for the selective vulnerability of these neurons and that the heavy subunit may have a crucial role in the disease process.

\section{Acknowledgments}

We would like to thank Heliomar Pereira Marcos and Suely Menezes da Cunha for excellent technical assistance and Suelen Adriani Marques for helpful suggestions regarding the immunohistochemistry technique.

\section{References}

1. Julien JP (2001). Amyotrophic lateral sclerosis: unfolding the toxicity of the misfolded. Cell, 104: 581-591.

2. Rowland LP \& Shneider NA (2001). Medical progress: amyotrophic lateral sclerosis. New England Journal of Medicine, 344: 1688-1700.
3. Talbot K (2002). Motor neuron disease. Postgraduate Medical Journal, 78: 513-519.

4. Lee MK \& Cleveland DW (1996). Neuronal intermediate filaments. Annual Review of Neuroscience, 19: 187-217. 
5. Schmidt ML, Carden MJ, Lee VM-Y \& Trojanowski JQ (1987). Phosphate dependent and independent neurofilament epitopes in the axonal swellings of patients with motor neuron disease and controls. Laboratory Investigation, 56: 282-294.

6. Louvel E, Hugon J \& Doble A (1997). Therapeutic advances in amyotrophic lateral sclerosis. Trends in Pharmacological Sciences, 18: 196-202.

7. Hirano A (1996). Neuropathology of ALS: an overview. Neurology, 47 (Suppl 2): 63-66.

8. Bredesen DE, Pazos W, Goto JJ, Rabizadeh S, Roe JA, Gralla EB, Ellerby LM \& Valentine JS (1996). Cell death mechanisms in ALS. Neurology, 47 (Suppl 2): 36-39.

9. Rosen DR, Siddique T, Patterson D, Figlewicz DA \& Sapp P (1993). Mutations in CuZn superoxide dismutase gene are associated with familial amyotrophic lateral sclerosis. Nature, 362: 59-62.

10. Carpenter S (1969). Proximal axonal enlargement in motor neuron disease. Neurology, 18: 841-851.

11. Hoffman PN, Griffin JW \& Price DL (1984). Control of axonal caliber by neurofilament transport. Journal of Cell Biology, 99: 705-714.

12. Hirokawa N, Glicksman MA \& Willard MB (1984). Organization of mammalian neurofilament polypeptides within the neuronal cytoskeleton. Journal of Cell Biology, 66: 351-366.

13. Napolitano EW, Chin SSM, Colman DR \& Liem RKH (1987). Complete amino acid sequence and in vitro expression of rat NF-M, the middle molecular weight neurofilament protein. Journal of Neuroscience, 7: 2590-2599.

14. Schlaepfer WW \& Freeman LA (1978). Neurofilament proteins of rat peripheral nerve and spinal cord. Journal of Cell Biology, 78 653-662.

15. Carden MJ, Schlaepfer WW \& Lee VM-Y (1985). The structure, biochemical properties, and immunogenicity of neurofilament peripheral regions are determined by phosphorylation state. Journal of Biological Chemistry, 260: 9805-9814.

16. Julien JP \& Mushynski WE (1982). Multiple phosphorylation sites in mammalian neurofilament polypeptides. Journal of Biological Chemistry, 257: 10467-10470.

17. Geisler N, Vandekerckhove J \& Weber K (1987). Location and sequence characterization of the major phosphorylation sites of the high molecular mass neurofilament proteins $M$ and $H$. FEBS Letters, 221: 403-407

18. Chen J, Nakata T, Zhizeng Z \& Hirokawa N (2000). The c-terminal tail domain of neurofilament protein-H (NF-H) forms the crossbridges and regulates neurofilament bundle formation. Journal of Cell Science, 113: 3861-3869.

19. Betts C, Blackstock WP, Ward MA \& Anderton BH (1997). Identification of phosphorylation sites on neurofilament proteins by nanoelectrospray mass spectrometry. Journal of Biological Chemistry, 272: 12922-12927.

20. Nixon RA, Paskevich PA, Sihag RK \& Thayer CY (1994). Phosphorylation on carboxyl terminus domains of neurofilament proteins in retinal ganglion cell neurons in vivo: influences on regional neurofilament accumulation, interneurofilament spacing, and axonal caliber. Journal of Cell Biology, 126: 1031-1046.

21. Tsang YM, Chiong F, Kuznetsov D, Kasarskis E \& Geula C (2000). Motor neurons are rich in non-phosphorylated neurofilaments: cross-species comparison and alterations in ALS. Brain Research, 861: 45-58
22. Inoué S \& Spring KR (1997). Video Microscopy. 2nd edn. Plenum Press, New York.

23. Fuchs E \& Cleveland DW (1998). A structural scaffolding of intermediate filaments in health and disease. Science, 279: 514-519.

24. Geisler N \& Weber K (1981). Self-assembly in vitro of the 68,000 molecular weight component of the mammalian neurofilament triplet proteins into intermediate-sized filaments. Journal of Molecular Biology, 151: 565-571.

25. Julien JP (1999). Neurofilament functions in health and disease. Current Opinion in Neurobiology, 9: 554-560.

26. Rouleau GA, Clarke AW, Rooke K, Pramatarova A, Krizus A, Suchowersky O, Julien JP \& Figlewicz DA (1996). SOD1 mutation is associated with accumulations of neurofilaments in amyotrophic lateral sclerosis. Annals of Neurology, 39: 128-131.

27. Xu Z, Marszalek JR, Lee MK, Wong PC, Folmer J, Crawford TO, Hsieh S-T, Griffin JW \& Cleveland DW (1996). Subunit composition of neurofilaments specifies axonal diameter. Journal of Cell Biology, 133: 1061-1069.

28. Kriz J, Meier J, Julien JP \& Padjen AL (2000). Altered ionic conductances in axons of transgenic mouse expressing the human neurofilament heavy gene: a mouse model of amyotrophic lateral sclerosis. Experimental Neurology, 163: 414-421.

29. Hedreen JC \& Koliatsos VE (1994). Phosphorylated neurofilaments in neuronal perikarya and dendrites in human brain following axonal damage. Journal of Neuropathology and Experimental Neurology, 53: 663-671.

30. Marques SA, Taffarel M \& Martinez AMB (2003). Participation of neurofilament proteins in axonal dark degeneration of rat's optic nerves. Brain Research, 969: 1-13.

31. Bajaj NPS, al-Sarraj ST, Leigh PN, Anderson V \& Miller CCJ (1999). Cyclin dependent kinase-5 (CDK-5) phosphorylates neurofilament heavy (NF-H) chain to generate epitopes for antibodies that label neurofilament accumulations in amyotrophic lateral sclerosis (ALS) and is present in affected motor neurones in ALS. Progress in Neuro-Psychopharmacology and Biological Psychiatry, 23: 833850

32. Ackerley S, Grierson AJ, Brownlees J, Thornhill P, Anderton BH, Leigh PN, Shaw CE \& Miller C (2000). Glutamate slows axonal transport of neurofilaments in transfected neurons. Journal of Cell Biology, 150: 165-176.

33. Chung KKK, Dawson VL \& Dawson TM (2001). The role of the ubiquitin-proteasomal pathway in Parkinson's disease and other neurodegenerative disorders. Trends in Neurosciences, 24 (Suppl 11): $7-14$

34. Alves-Rodrigues A, Gregori L \& Pereira MEF (1998). Ubiquitin, cellular inclusions and their role in neurodegeneration. Trends in Neurosciences, 21: 516-520.

35. Migheli A, Pezzulo T, Attanasio A \& Schiffer D (1993). Peripherin immunoreactive structures in amyotrophic lateral sclerosis. Laboratory Investigation, 68: 185-191.

36. Deslisle MB \& Carpenter S (1984). Neurofibrillary axonal swellings and amyotrophic lateral sclerosis. Journal of the Neurological Sciences, 63: 241-250.

37. Toyoshima I, Yamamoto A, Masamune O \& Satake M (1989). Phosphorylation of neurofilament proteins and localization of axonal swellings in motor neuron disease. Journal of the Neurological Sciences, 89: 269-277. 